\title{
Multilevel Compound Tree - Construction Visualization and Interaction
}

\author{
François Boutin ${ }^{1}$, Jérôme Thièvre ${ }^{2}$, and Mountaz Hascoët ${ }^{3}$ \\ ${ }^{1}$ LIRMM, CNRS, Montpellier \\ francois.boutin@univ-montp1.fr \\ ${ }^{2}$ INA, Paris \\ jthievreaina.fr \\ ${ }^{3}$ LIRMM - CNRS, Montpellier \\ mountaz@lirmm.fr
}

\begin{abstract}
Several hierarchical clustering techniques have been proposed to visualize large graphs, but fewer solutions suggest a focus based approach. We propose a multilevel clustering technique that produces in linear time a contextual clustered view depending on a user-focus. We get a tree of clusters where each cluster - called meta-silhouette - is itself hierarchically clustered into an inclusion tree of silhouettes. Resulting Multilevel Silhouette Tree (MuSi-Tree) has a specific structure called multilevel compound tree. This work builds upon previous work on a compound tree structure called MO-Tree. The work presented in this paper is a major improvement over previous work by (1) defining multilevel compound tree as a more generic structure, (2) proposing original space-filling visualization techniques to display it, (3) defining relevant interaction model based on both focus changes and graph filtering techniques and (4) reporting from case studies in various fields: co-citation graphs, relateddocument graphs and social graphs.
\end{abstract}

\section{Introduction}

Graph clustering objective is to minimize inter-connectivity (edges between clusters) and maximize intra-connectivity (edges inside clusters). A multilevel clustering technique is used to organize large graphs [5][7]. It provides a hierarchical clustered graph that consists of a graph of clusters where each cluster is itself hierarchically clustered. If we induce edges between clusters, we get a structure called compound graph (section 2.2) [16].

We are interested in multilevel clustering techniques that produce contextual graph views from any focus. The idea is to be able to organize information "around" a focus using graph connectivity.

Previous work on MO-Tree [3] introduced a focus based multilevel clustering technique that provides a compound tree structure (section 2.2). It is a tree of clusters, where each cluster is itself hierarchically clustered. For instance, considering a connected graph in Fig.1, a MO-Tree view from focus 1 is displayed in Fig.2.

In this paper we define multilevel compound tree which improves the concept of compound tree. We also provide an algorithm for the construction of such structure. 
We further propose a space-filling visualization technique and an interaction model for displaying and interacting with this structure. Moreover, we report results of three case studies using co-citation graphs, related-document graphs and social graphs.

In the next section, we present a synthesis of related work on graph clustering, and multilevel visualization/interaction techniques. Section 3 further describes the new multilevel compound tree structure called MuSi-Tree and its construction algorithm. In section 4, we propose space-filling visualisation techniques to display this structure and an associated interaction model. Finally, in section 5, we report the results of three case-studies that aim at:

- Producing real graphs visualization and interaction from various perspectives.

- Applying filtering techniques to produce understandable MuSi-Tree structures.

- Providing qualitative results from a set of 56 users (section 5.4).
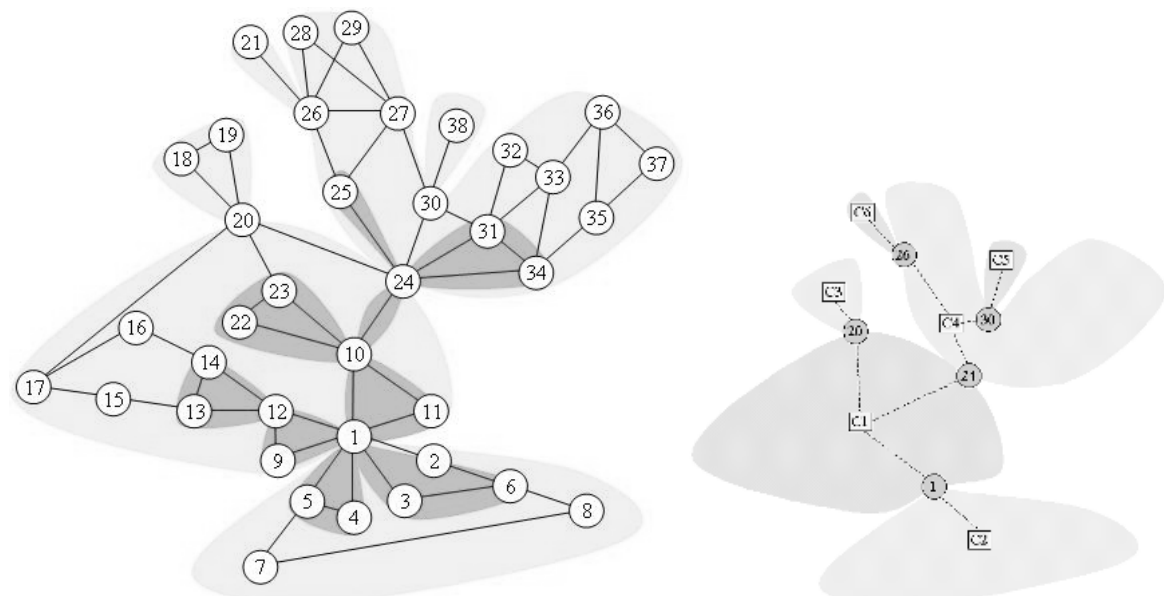

Fig. 1. (a) Graph clustering into silhouettes, (b) silhouette tree

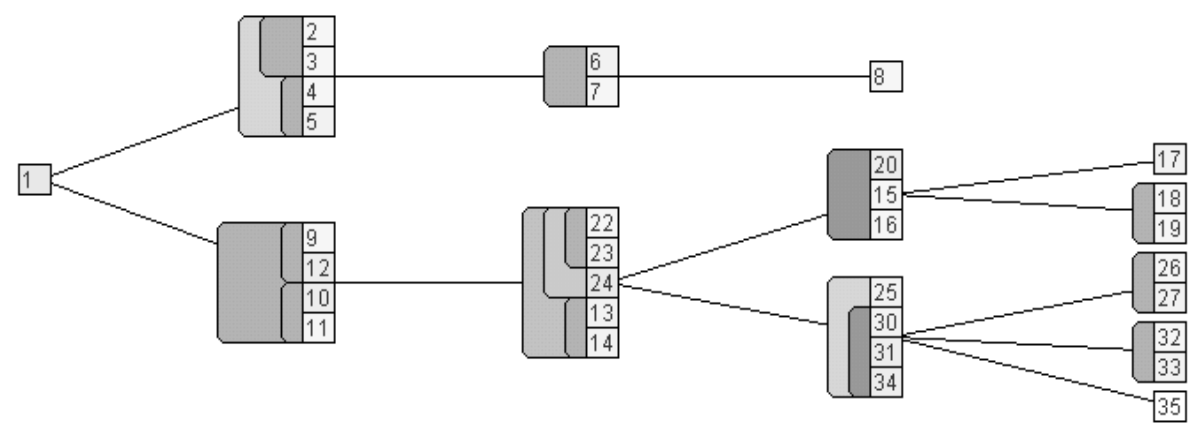

Fig. 2. MO-Tree from focus 1 


\section{Related Work}

\subsection{Graph Partitioning Techniques - A Review}

There are different ways to classify partitioning techniques [1][6][8]. We propose main criteria and illustrate them with some methods (see table 1).

Firstly, geometrical techniques take into account nodes coordinates: for instance, $K$-Means [1][11] is a local clustering method that produces iteratively a k-partition using centroïds. Global geometric methods [6][8][13] are based on energy models, inertial algorithms or graph bisection (with hyperplans or spheres).

Secondly, structural algorithms are based on graph connectivity. The principle of a local clustering technique is to minimize inter cluster connectivity using nodes exchange, agglomeration or separation. Global structural [1][4][6][8] techniques use matrix operations: Markov clustering is a stochastic method based on flow computing while spectral techniques consist in mapping nodes into an eigenspace. Spectral techniques may be used to provide coordinates for geometric clustering methods.

Many algorithms [11][13] use both global and local methods to refine clustering. Choice of clustering technique may depend on graph propriety (size, connectivity...), but also clustering constraints (balanced clustering, edge crossing minimization...).

Table 1. clustering criteria and methods

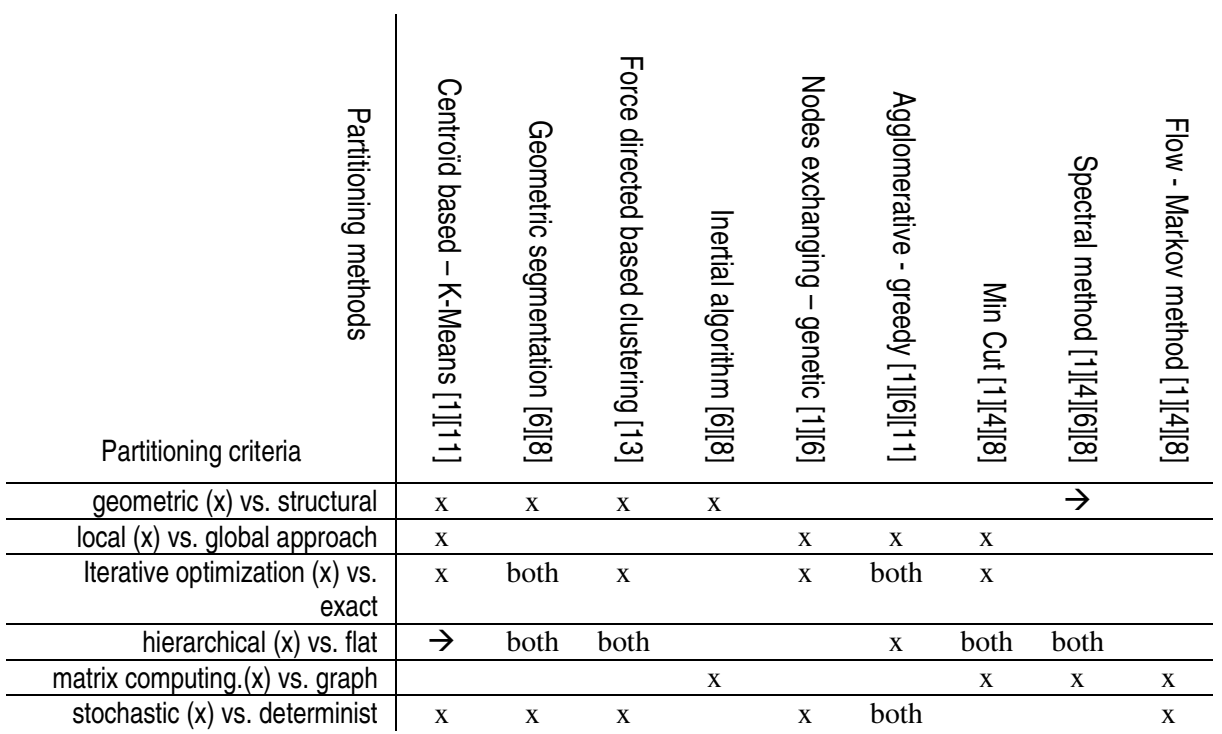

Multilevel clustering techniques were developed to organize large graphs into hierarchical clustered graph (section 2.2). For that purpose, some "flat" techniques are adapted. For instance $K$-Means can be applied recursively [11] (see $\rightarrow$ in Table 1). Cutting techniques depending on a threshold may be computed with many thresholds.

Otherwise, hierarchical clustering techniques proceeds iteratively with merging or splitting the most appropriate clusters according some metric. Resulting dendrogram can be cut at different levels to provide a hierarchical clustered graph [1][11]. 
According to our criteria, our method is a structural-local-exact-hierarchical-graphbased-determinist clustering technique.

\subsection{Multiscale Structure - Definitions and Visualization}

Now, we review and define new multilevel graph structures:

- A hierarchical clustered graph was defined [5] by a graph $G=(V, E)$ and a rooted tree T. Leaves of T are vertices of $G$. Other nodes in T are sets of nodes of $G$ called clusters. $\mathrm{T}$ describes an inclusion relation between clusters so $\mathrm{T}$ is called inclusion tree. $2 D$ and $3 D$ views (see Fig.3) are proposed in [7]: inclusion tree $\mathrm{T}$ is described either by inclusion areas (Fig.3.a) or dotted arrows (Fig.3.b).

- A compound graph consists of a hierarchical clustered graph with edges between clusters [16]. 2D and $3 D$ views are proposed in [5][7] (Fig.3.a,b). Edges between clusters are drawn in thin lines for small clusters and thick lines for large clusters: their removal induces a hierarchical clustered graph.

- We defined in [3] a compound tree as a compound graph with an adjacency tree of meta-clusters (largest clusters). For instance, (Fig.3.c) presents a compound tree since largest clusters belong to an adjacency tree. We proposed in [3] flat compound tree visualization (Fig.2) of G (Fig.1): to avoid a cluttered view, edges between meta-clusters are displayed unlike edges between clusters into meta-clusters.

- In this paper, we define a multilevel compound tree as a compound tree where each layer consists of an adjacency tree of clusters. For instance (Fig.3.d) meta-clusters but also small clusters belong to adjacency trees. We propose an algorithm that computes a specific multilevel compound tree structure called MuSi-Tree. This structure is general enough to be provided by other clustering techniques.

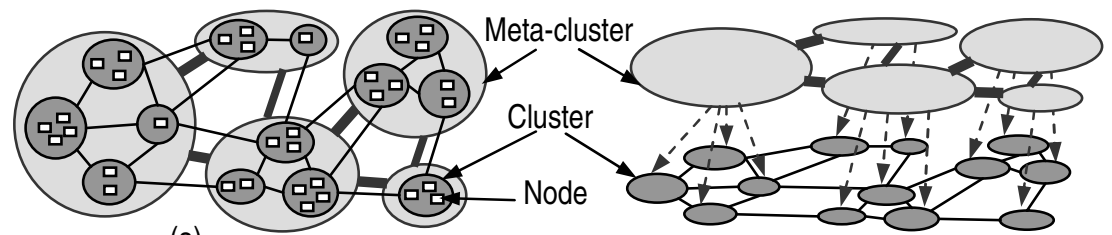

(a)

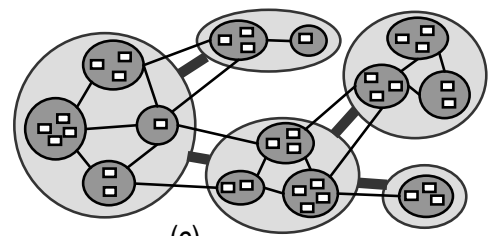

(c)

(b)

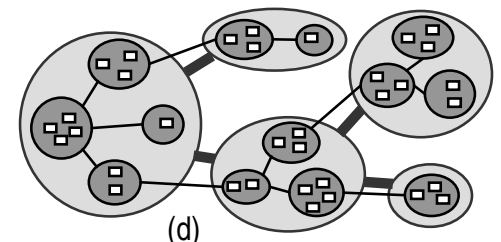

(d)

Fig. 3. (a) \& (b) compound graph, (c) compound tree, (d) multilevel compound tree

\subsection{Visualization and Interaction Concepts - Multilevel Approach}

$2 D$ and $3 D$ views of hierarchical clustered graphs based on tree layouts are described in [5] and [7] respectively. These visualizations support multilevel views by allowing 
user to choose level of visibility of cluster hierarchy, or manually collapse/expand clusters. Fisheye and full zoom methods are compared in [14].

On the other hand, the use of upgraded force-directed models to layout clustered graphs has been studied in [10][17]. The main idea here is to use different forces for intra and inter clusters links and add an invisible attractor vertex to force vertices of the same cluster to keep close to each other.

Yee and al. propose a focus-based radial tree layout of non-clustered graphs [18]. The system builds a breadth-first spanning tree of the graph from a focus node selected by the user. The focus node is laid out at the centre of the display and the others nodes are located on concentric rings corresponding to their shortest distance to the focus. Angular position of a node depends on its parent position in the spanning tree. Angular width of each node is computed from the angle needed by its subtree. This system presents a particularly interesting work on animation of focus changes. When the user selects another focus, all nodes move to their new position, following an intuitive path with a smooth slow-in, slow-out timing. The prefuse toolkit [9] offers a smart implementation of this technique. Nevertheless, as mentioned earlier, neither particular visualization nor specific interactions have been implemented for multilevel data structures.

In this work we propose an add-on to prefuse [9] that supports the visualization of multilevel compound trees (Section 4). This technique is used with MuSi-trees that are produced from our clustering technique. A MuSi-Tree consists of a particular case of multilevel compound tree. Nevertheless our visualization technique is not limited to MuSi-tree. It can be used for any type of multilevel compound tree. Since multilevel compound tree structure is general enough, we believe that this technique can be used to visualize other clustering techniques results.

\section{Multilevel Graph Clustering Technique}

\subsection{Main Principles and Definitions}

Let us consider a connected graph $G=(V, E)$. A node is called articulation node if its removal disconnects $G$. Splitting (but not removing) articulation nodes disconnects $G$ into maximal biconnected components or trivial components (two connected nodes). Indeed, any non trivial component is biconnected else it would be split up into components. Maximal biconnected or trivial components are called silhouettes.

Graph $G$ is a bipartite tree that connects articulation nodes and silhouettes, see (Fig.1.b). Indeed, if there was a cycle between silhouettes, then silhouettes should belong to a same component, what is impossible since they are maximal.

The silhouette tree decomposition can be applied to graph $G$ but also to any connected sub graph $G$ ' of $G$. Considering a silhouette of $G$ ', it naturally belongs to one silhouette of $G$. Consequently, we say that silhouette tree of $G$ ' belongs to silhouette tree of $G$. In Fig.1.a, we present two silhouette trees: one for graph $G$, another one for a connected sub graph $G^{\prime}$ (see darkest included areas).

Now, the purpose is to be able to choose interesting sub graphs of $G$, in order to apply our algorithm recursively. We present our approach in section 3.2. 


\subsection{Focus Based Clustering Approach}

We propose a clustering technique that takes into account a focus-node. So, we get different graph perspectives depending on the focus we select. Resulting computing is very efficient as explained in section 3.3.

Nodes are displayed into $K_{\max }$ levels according to their distance to the focus. $G_{k}$ is defined as the connected sub-graph of $G$ that contains nodes at distance at most $k$ from the focus. Sil $k_{k}$ is defined as the silhouette tree of $G_{k}$. The set of silhouettes trees $\left\{S i l_{k}\right\}$ is ordered with inclusion relation. It means that $\mathrm{Sil}_{k}$ includes $\mathrm{Sil}_{i}$ if $k$ is above $i$.

$\left\{G_{k}\right\}$ is computed in linear time using a breadth-first search. Sil $l_{k+1}$ is also naturally computed from $\operatorname{Sil}_{k}$ (section 3.3) and resulting computing of $\left\{\mathrm{Sil}_{k}\right\}$ is linear. The set of silhouettes $\left\{\mathrm{Sil}_{k}, 0 \leq k \leq K_{\max }\right\}$ is called Multilevel Silhouette Tree (MuSi-Tree).

$\mathrm{Sil}_{\mathrm{Kmax}}$ is called meta-silhouette tree. It is a tree of meta-silhouettes. Each metasilhouette is itself an inclusion tree of silhouettes.

\subsection{Optimized Algorithm}

Let consider graph $\mathrm{G}$ organized into $\mathrm{K}_{\max }$ levels from a focus node. We describe our MuSi-Tree optimized algorithm (Fig.4). Sil $l_{0}=\{f o c u s\}$. Sil $k_{k+1}$ is computed using Sil $l_{k}$ :

- Level $k+1$ is first organized into connected components. See Fig.4, resulting connected components: $\{a b\},\{c d\},\{e\},\{f\},\{g\},\{h i j\},\{k\},\{l m\},\{n o\}$.

- If a connected component is related to only one node $v$ on level $k, v$ is an articulation node for $\mathrm{Sil}_{k+1}$ that connects a new silhouette. For instance, see Fig.4, $\{a b\}$, $\{c d\},\{e\},\{f\},\{g\},\{l m\}$ belong to new silhouettes.

- Else a large silhouette is created that includes silhouettes of $S i l_{k}$ connected by this connected component. See Fig.4: components $\{h i j\},\{k\},\{n o\}$

So, the resulting MuSi-Tree structure is computed in linear time.

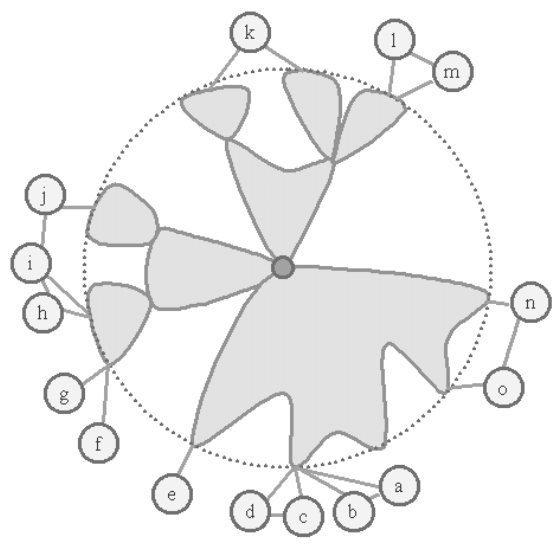

level $\mathrm{k}$

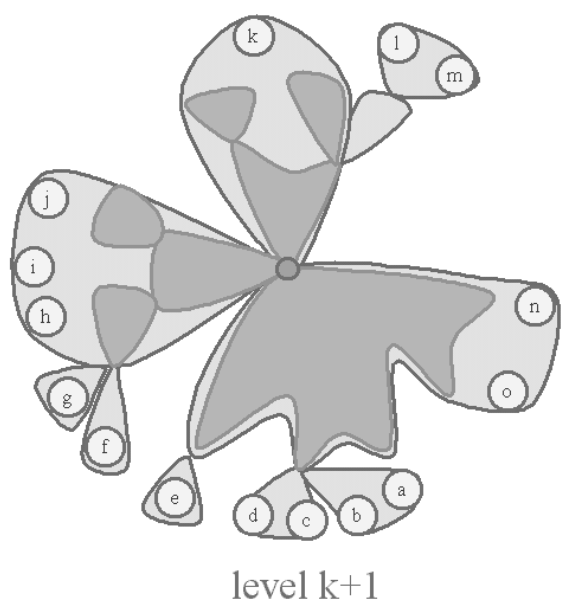

level $\mathrm{k}+1$

Fig. 4. Graph clustering algorithm - step k+1 


\subsection{Invariant Sets}

We can get various MuSi-Trees depending on the focus we take. However, metasilhouette tree structure remains the same. Indeed, global articulation nodes and metasilhouettes are graph invariants. Focus changes will be used in the interaction model and will benefit this property as explained in the next section.

\section{MuSi-Tree Visualization and Interaction}

\subsection{MuSi-Tree Layout}

The nature of our clustering, based on a focus node and distances between nodes and this focus, leads us to choose a hierarchical layout. Among existing hierarchical layout, radial layout is really well-suited. Nodes are located on concentric rings around focus, which makes distance between each node and the focus explicit [18]. Moreover, radial layout makes a better use of screen space than classical top-down tree layout.

Our layout is quite similar to radial layout [18]. Our main contribution is in the drawing of silhouettes. To perform this, we started by transforming MuSi-Tree into a well-suited spanning tree. This mainly implies particular ordering of spanning tree nodes to ensure (1) silhouettes graphical cohesiveness and (2) non-overlapping. Once nodes locations have been computed with respect to ordering constraint, we perform a depth-first traversal and draw each silhouette as the bounding shape of its nodes.

Initial graph (Fig.5.a) is iteratively clustered by level into meta-nodes (light areas Fig.5.b) using a former technique introduced in [3]. Each silhouette is computed as a tree of meta-nodes (Fig.5.c). Geometric outline of this tree generates the drawing of silhouette (dark areas).

We attribute different colors to silhouettes trees to easily recognize non trivial biconnected components in the graph. Each hierarchy of silhouettes is attached to an articulation node that is painted with the same color. Articulation nodes are not included in their corresponding silhouette to avoid multiple overlaps that would make the visualization too complex. The use of transparent colors to fill silhouettes helps to perceive their inclusion level: the deeper they are, the darker they look.

Visualization is built over the prefuse framework [9] that provides support for animated transformations on graph layout. It is used to animate our focus changes.

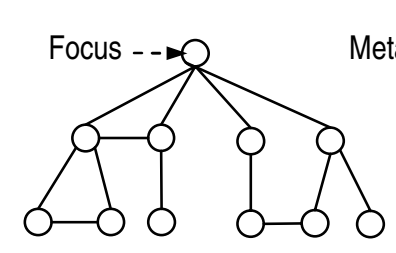

(a)

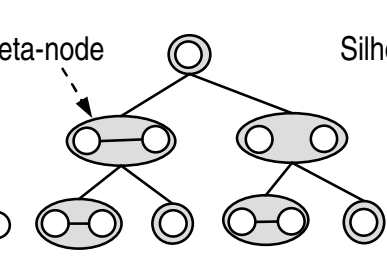

(b)

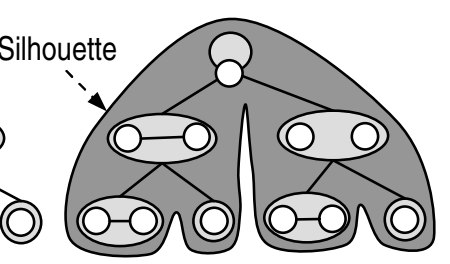

(c)

Fig. 5. (a) Initial graph: hierarchical view (b) clustering by level (c) silhouette drawing 


\subsection{Changing Focus}

Transitions between focus changes are animated, to help the user in tracking objects of interest and keeping a coherent perception of graph structure [18]. At this moment, only nodes and links are animated. The silhouettes are not displayed during transition because it is quite hard to get smooth animation of their transformation since their shape can change completely between two states, but we keep working on this interesting problem.

\subsection{Dynamic Filtering}

Graph visualizations provide great information on data structures and relations, and some properties can be displayed by using visual attributes like colours, size and shape. However, it is very hard to show the complete information attached to a node inside the graph view, and it is especially true for textual information.

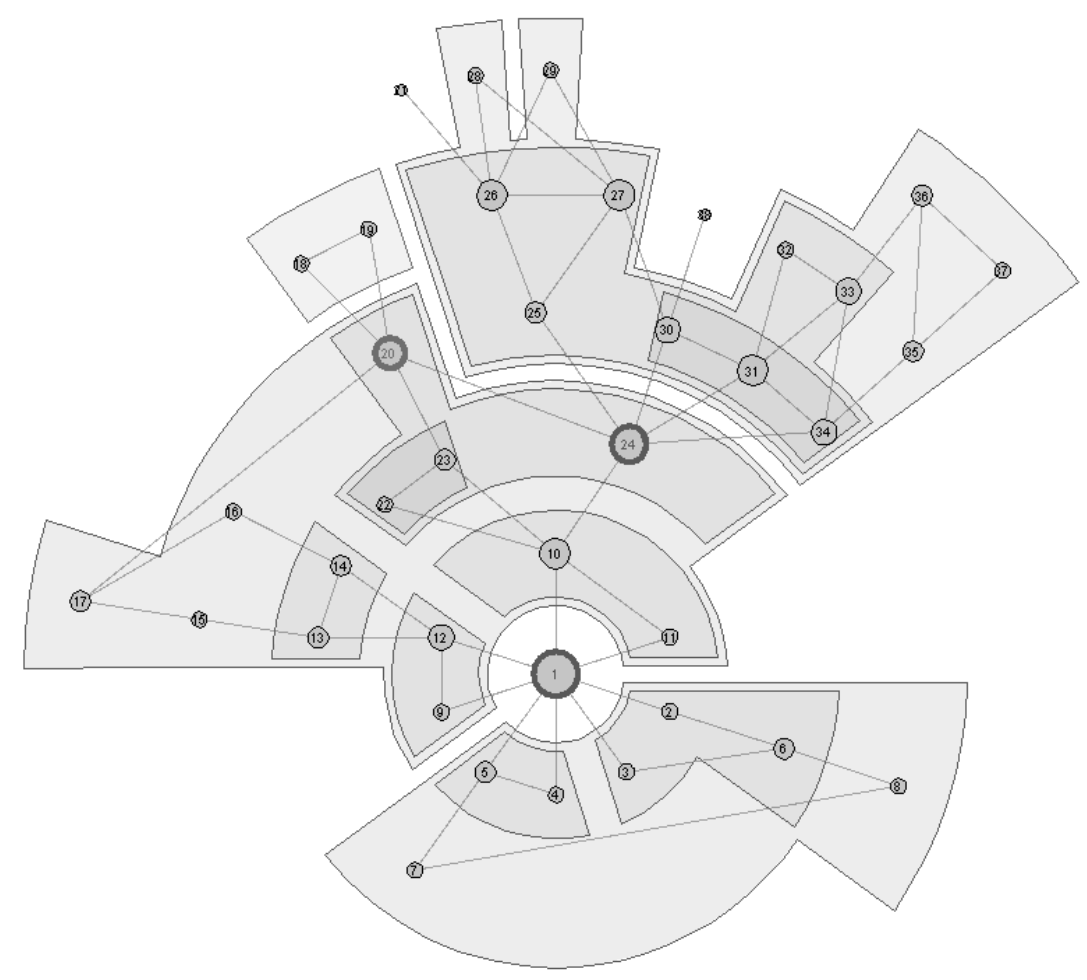

Fig. 6. Multilevel Silhouette Tree (MuSi-Tree) from focus 1

To solve this problem, we use a traditional data table to display nodes and their attributes. This widget supports multiple sorts and filters. Combination of these two features allows users to perform quick searches and selections over the whole dataset. We propose different filtering features depending of nodes and edges attributes types: 
- Regular expressions for textual attributes,

- Minimum and maximum threshold for numeric attributes,

- Temporal intervals for dates.

Filtering is easily reversible and its effects (dynamic insertions, nodes and links removals) are animated to offers users a good perception of changes.

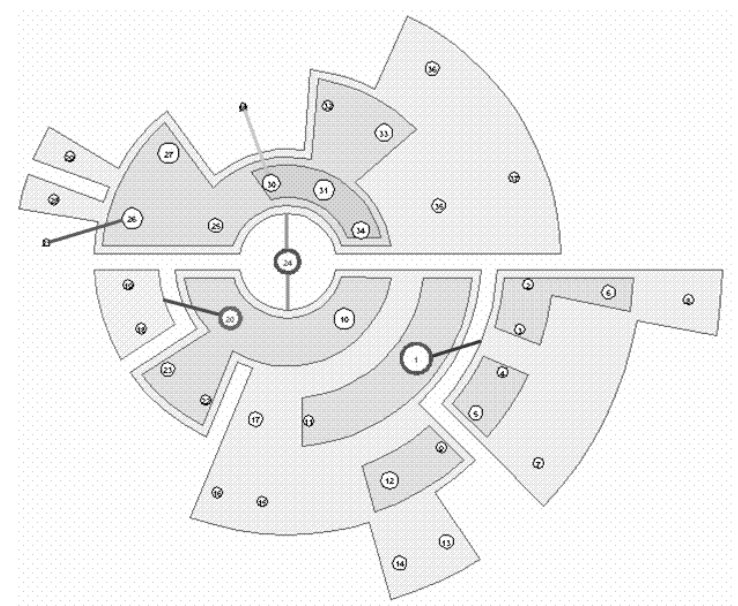

Fig. 7. Multilevel Silhouette Tree (MuSi-Tree) - compact view from focus 24

\section{Case Studies}

\subsection{Citation Graph}

We have clustered a connected citation graph including 122 papers and 206 links. It was collected on Research Index (CiteSeer) [12] from focus: "Navigation and interaction within graphical bookmarks" (Fig.8). Three large silhouettes are related to: information visualization, database visualization and document classification (Fig.9). All silhouettes and articulation nodes belong to a silhouette tree (Fig.9).

\subsection{Related-Document Graph}

The aim is to propose a map of conferences "around" Interact 2005. For that purpose, we use TouchGraph GoogleBrowser [15] that iteratively produces a spring view of 101 conferences related to www.interact2005.org (Fig.10.b). To manage a well connected graph, we consider nodes with 2 or more incoming edges. We get 200 edges.

We present MuSi-Trees from two foci: www.interact2005.org (Fig.10.a) and NYC 2004: www2004.org (Fig.11). Resulting views share five non trivial silhouettes that represent five specific domains: visualization/interaction, ergonomics, computational linguistic, digital library and image processing. We get different articulation nodes between domains: NYC 2004 and SIGIR 2004 introduce digital library and computational linguistic. VIS 2005 connects visualization/interaction with image processing. HCII 2005 is a bridge between visualization/interaction and ergonomic. 


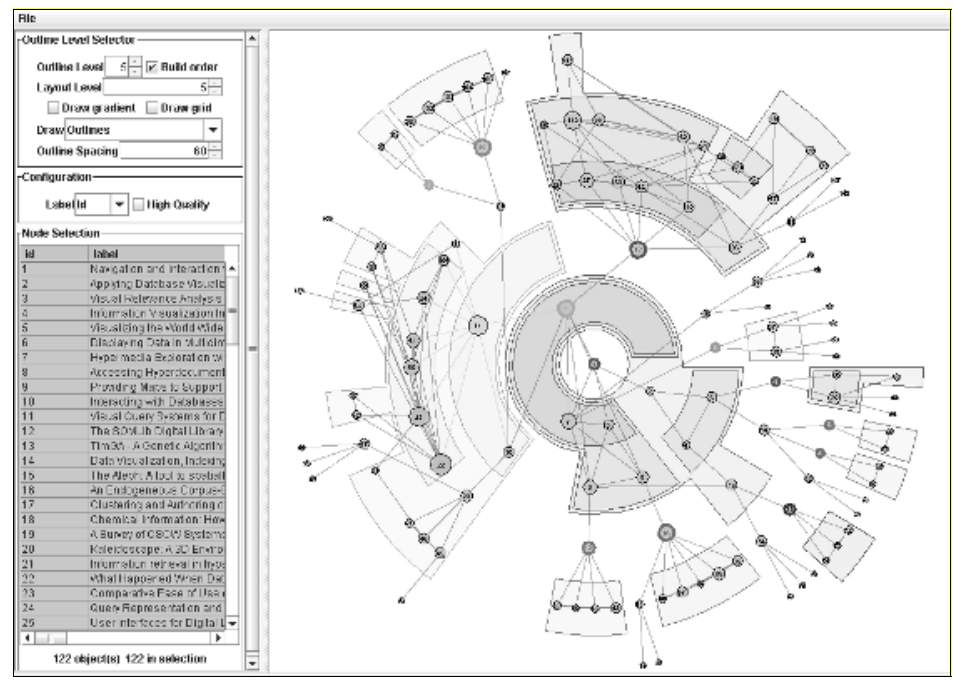

Fig. 8. MuSi-Tree Viewer - citation graph clustering

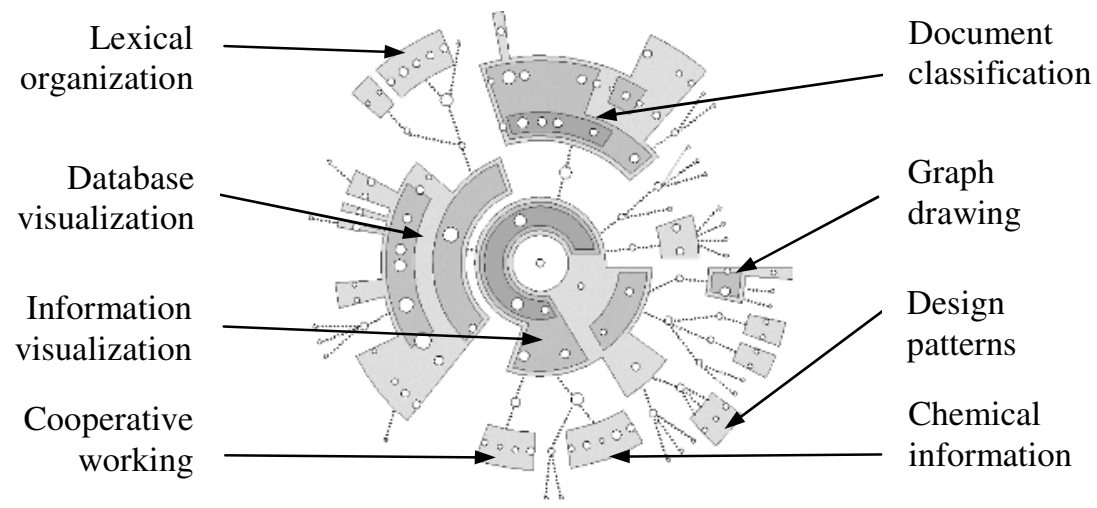

Fig. 9. MuSi-Tree - compact view

\subsection{Social Graph}

In this study, 56 medical students divided into four work groups were asked about their friendship relations (strong or weak). To begin with, whole social graph including all relations (unilateral and bilateral, strong and weak) is displayed in Fig.12. We get a single well connected component.

Then, a filtering algorithm is applied to include bilateral strong relations. Social graph is displayed from the same focus (Fig.13). We denote one large component in relation with four smaller components. In fact, each small group belongs more or less to a work group. Five students create relations between these groups. 

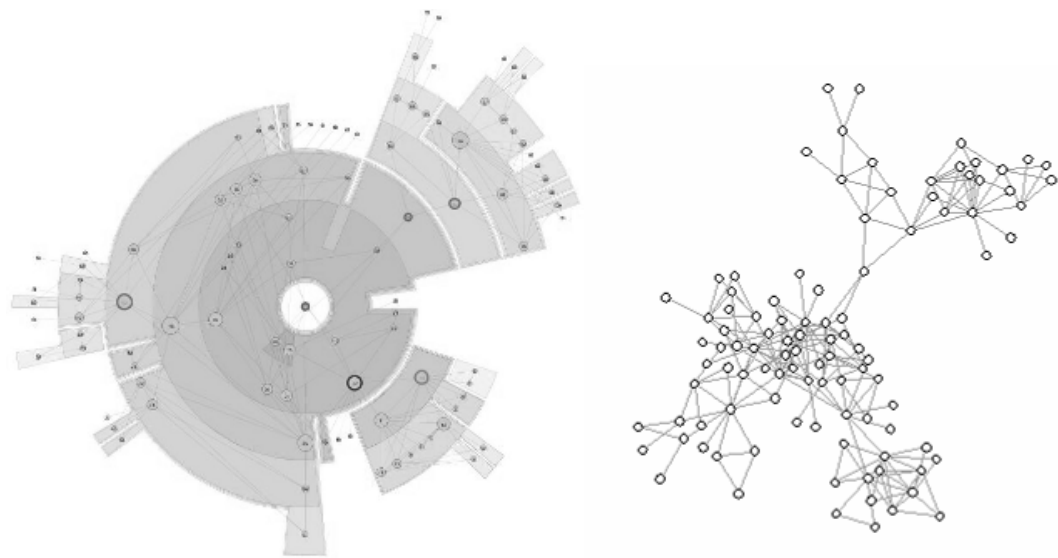

Fig. 10. (a) MuSi-tree from focus "Interact 2005", (b) TouchGraph view [15]

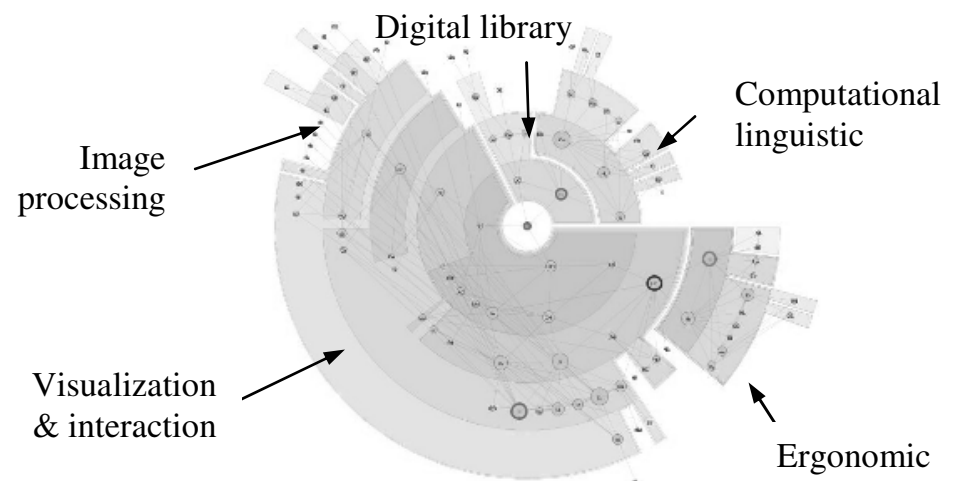

Fig. 11. Organization from focus: "NYC 2004"

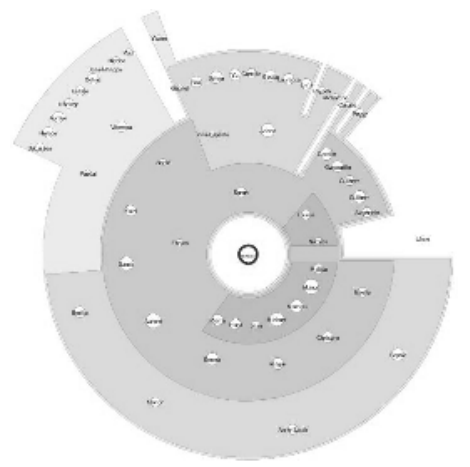

Fig. 12. Whole relations between students 


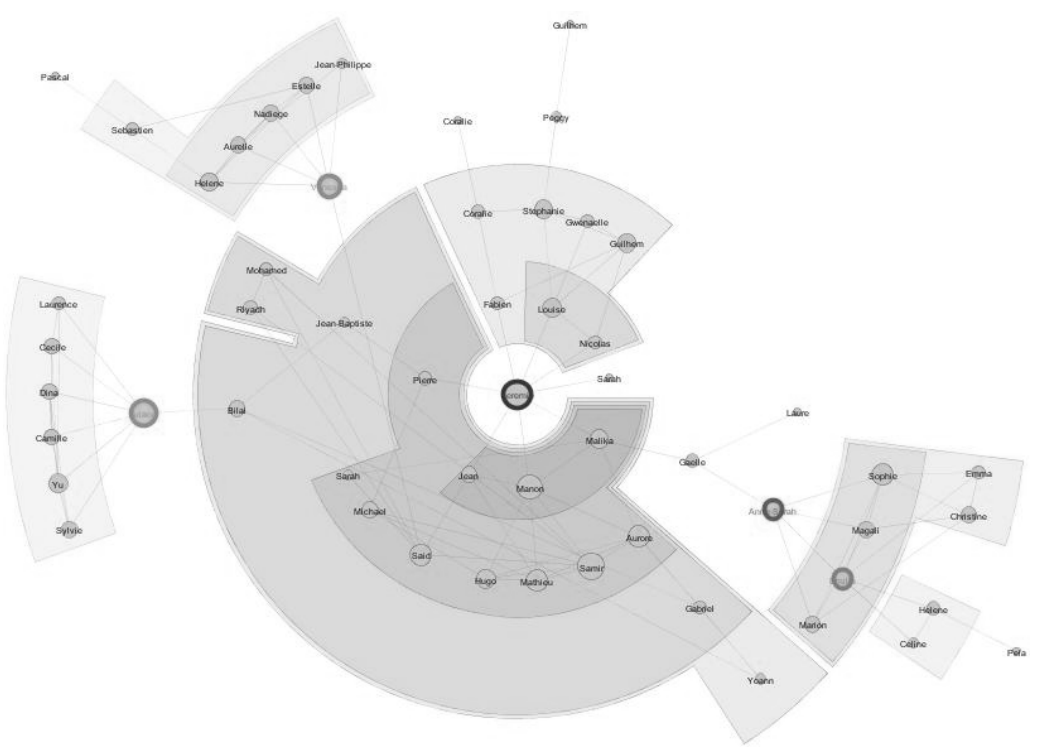

Fig. 13. Strong bilateral relations between students

\subsection{Qualitative Evaluation}

After collecting data, resulting social MuSi-Trees were presented to the four groups of medical students (without experience in information visualization). A five minutes show included visualization and interactive techniques: focus changing and group zooming. MuSi-Tree was presented as a "strong friendship map" around a student. No more explanation about the clustering algorithm was given. Students were invited to express themselves about their feeling on the clustering results, and the visualization/ interaction technique. The study reveals qualitative results:

- Majority of students considered the map as meaningful, even though they knew nothing about clustering technique.

- They recognized many natural groups of friends.

- They naturally understood dark areas as cohesive groups.

- Students were interested in changing focus to draw their own friendship map. They sometimes felt lost especially when focus moved from a silhouette to another.

- Students had sometimes difficulties to follow nodes when the graph was moving. Nevertheless, they often recognized silhouettes from their shape or their color.

This preliminary study invites us to practice a quantitative study to compare both our clustering technique, and our visualization/interaction technique with other ones.

\section{Conclusion}

In this paper we proposed a focus-based multilevel agglomerative technique. Resulting structure called Multilevel Silhouette Tree (MuSi-Tree) is easy to explore and easy to manage because of its features (multilevel compound tree). 
We applied our algorithm to a citation graph, a related-document graph and a social graph. Citation graph was displayed in a compact view that reveals silhouette tree structure. We present a graph of related sites displayed from two foci. Resulting views share meta-silhouettes and articulation nodes. Applying filtering techniques help the user understanding clustering structure.

MuSi-Tree appeared to be particularly well suited to organize a locally well articulated graph. It consists of a graph with "some" articulation nodes in its various kneighbourhood. It will be interesting to study locally well articulated graph indices. In order to improve visualisation of large graphs with high connectivity we propose to use filtering techniques in a pre-processing stage of our clustering technique. The objective will be to extract a "nice" multilevel silhouette tree structure.

More intensive evaluation is needed to better clarify conditions in which our clustering and visualization techniques can best benefit users interacting with large graphs. The question of what criteria should be used for the evaluation of clustering and visualization results is an open issue. A good evaluation of this work can only be a long and challenging task. We have already conducted many informal evaluations and studies on this work, but we are now deeply engaged into a more controlled evaluation approach. In this area, we plan to perform larger evaluation both in terms of analytical criteria [2] as in terms of controlled experiments involving real users.

\section{References}

1. Alpert C.J. and Kahng A.B. Recent Developments in Netlist Partitioning: A Survey, Integration: the VLSI Journal, vol. 19, pp. 1-81, 1995.

2. Boutin F. and Hascoët M., Cluster Validity Indices for Graph Partitioning, Proceedings of the Conference on Information Visualization IV'2004.

3. Boutin F. and Hascoët M., Focus Dependent Multi-level Graph Clustering. Proceedings of the Conference on Advanced Visual Interfaces, AVI 2004, ACM.

4. Brandes U., Gaertler M., and Wagner D.: Experiments on Graph Clustering Algorithms. Proc. ESA '03, LNCS 2832, pp. 568-579. (c) Springer-Verlag, 2003.

5. Brockenauer R. and Cornelsen S., Drawing Clusters and Hierarchies. In Michael Vaufmann and Dorothea Wagner (Eds.): Drawing Graphs: Methods and Models, LNCS 2025, pp. 194 - 228. (C) Springer-Verlag, 2001.

6. Chamberlain B.L. Graph Partitioning Algorithms for Distributing Workloads of Parallel Computations. Technical Report UW-CSE-98-10-03, Univ. of Washington, 1998.

7. Eades P., Multilevel Visualization of Clustered Graphs, Proceedings of Graph Drawing'96, Berkeley, California, September, 1996.

8. Elsner U. 1997. Graph partitioning - A survey. Technical Report 393, Technische Universitat Chemnitz.

9. Heer J., Card S.K., and Landay J.A., prefuse: a toolkit for interactive information visualization. In CHI 2005, Human Factors in Computing Systems, 2005.

10. Huang M. L., Eades P. A Fully Animated Interactive System for Clustering and Navigating Huge Graphs, Proceedings of the 6th International Symposium on Graph Drawing (GD'98), Springer LNCS 1547, pages 374-383, 1998.

11. Karypis G., Han E-H and Kumar V. CHAMELEON: A hierarchical clustering algorithm using dynamic modeling. IEEE Computer, 32(8): 68-75, 1999.

12. Lee Giles C., Bollacker K.D., Lawrence S.: CiteSeer: An Automatic Citation Indexing System. ACM DL 1998: 89-98. 
13. Noack A. An energy model for visual graph clustering. In G. Liotta, editor, Proceedings of GD 2003, LNCS 2912, pages 425--436, Berlin, 2004. Springer-Verlag.

14. Schaffer D., Zhenping Z., Grreenberg, S. et al. Navigating Hierarchically Clustered Networks through Fisheye and Full-Zoom Methods, ACM Transactions on Computer-Human Interaction volume 3-2, pages 162-188, 1998.

15. Shapiro A., TouchGraph, Dynamic Graph Layout Tool, www.touchgraph.com

16. Sugiyama K., Tagawa S. and Toda M. Methods for Visual Understanding of Hierarchical System Structures. IEEE Transactions on Systems Man and Cybernetics, 1981.

17. Wang X., Miyamoto I. Generating Customized Layouts, Proceeding of the 3rd International Symposium on Graph Drawing (GD'95), Springer LNCS 1027, 504-515, 1995.

18. Yee K.P., Fisher D., Dhamija R. \& Hearst M.A. Animated Exploration of Dynamic Graphs with Radial Layout. Proceeding of IEEE Symposium on Information Visualization, 2001. 